\title{
Evaluating Mobile Phones as Risk Information Providers
}

\author{
Stephan von Watzdorf, Florian Michahelles \\ ETH Zurich \\ Scheuchzerstrasse 7 \\ $\mathrm{CH}-8092$ Zürich, Switzerland \\ \{swatzdorf, fmichahelles\}@ethz.ch
}

\begin{abstract}
Information about health, disease or environmental conditions is increasingly becoming available. We investigate the suitability of using mobile phones as an interface to provide information about risk-related events or conditions to the user. We approach the problem by conducting an online survey in order to match the requirements on a risk information service with the capabilities of the mobile phone and to evaluate different notification mechanisms, the usage frequency, and the influence of costs. Based on the results of the survey we confirm the suitability of the mobile phone to provide risk-related information as well as the user's willingness to pay for the service.
\end{abstract}

\section{Categories and Subject Descriptors}

H.5.2 [Information Interfaces and Presentation]: User Interfaces - Evaluation/methodology, Prototyping. H.1.2 [Computer Applications]: Administrative Data Processing Business, Marketing.

\section{General Terms}

Design, Management, Experimentation.

\section{Keywords}

Risk Information, Mobile Phone, Technology-Acceptance Study, Smart-Alerts.

\section{INTRODUCTION}

With the emergence of pervasive computing devices and sensor networks environmental, and health-related data is being captured at an increasingly detailed level and broadcasted through the World Wide Web. While the MIT initiative healthmap.org is providing details about global diseases the UK FloodNet project [1] uses a grid network of sensors to predict floods. Similarly, the Austrian Federal Environment Agency and the SF Meteo weather service employ sensor data in order to detect elevated ozone values or hailstorms and notify citizens via SMS.

The provision of such risk-related information is not only an opportunity for authorities to inform citizens about severe environmental or health-related conditions. Notification systems about extreme weather conditions or health issues in foreign countries can e.g. be offered by insurance providers or travel agencies as value-added services. With the data about risk-related events or conditions at hand all those providers now face the

Copyright is held by the author/owner(s).

MobileHCI'09, September 15 - 18, 2009, Bonn, Germany.

ACM 978-1-60558-281-8. problem of finding a suitable interface in order to distribute the information to the end-user.

First efforts have been made here by employing the mobile phone. Besides the possibility of informing the users via SMS, further examples can be found where mobile phones have been used as an interface supporting the user's daily routine with selective risk information [2] or providing avalanche warnings for off-piste skiing [3]. The benefits of using mobile phones as an interface to provide information have been investigated before, e.g. in [4], where non-technical and technical advantages such as the wide market penetration and infrastructure of the mobile phone have been discussed.

Although the mobile phone is considered a mature technology, insights in the suitability of the mobile phone as an interface to provide risk-related information are scarce. When investigating the usefulness and suitability of a single service on the mobile phone two major approaches are proposed in the literature mostly dependent on the maturity of the employed technology. While mature technologies and established applications are being evaluated with technology acceptance studies [5] less ripe solutions are analyzed with practical user studies [6]. While few examples can be found where services that provide risk-related information have been evaluated in field studies [7], the analysis of the suitability and usability based on technology acceptance studies is still pending:

We thus investigate the suitability of the mobile phone as an interface to provide risk-related information. (1) Firstly we address the problem on a conceptual level in order to match the requirements on a risk information service with the capabilities of the mobile phone. (2) Secondly, we evaluate different notification mechanisms. (3) Finally, we investigate the user's intention to use a risk information service provided through a mobile phone as well as the usage frequency and the influence of costs.

The paper is organized as follows: Section two discusses related work and section three the design and proceeding of the user acceptance study. The results of the survey are described in section four and finally discussed in section five. We conclude and describe future research challenges in chapter 6 .

\section{RELATED WORK}

When considering the provision of information on mobile phones and the accompanying questions of evaluating the use of it, we can revert to former work from a methodological perspective. In order to reach our goal of investigating the suitability and usability of a mobile service we employ extensions to the Technology Acceptance Model (TAM) [8] to assess the usability, 
the user's intention to use the service, as well as the influence of costs.

Besides the investigation of related work from a methodological perspective, existing contributions can be found which considered the idea of providing risk-related information using the mobile phone as an interface [2], [3]. Other than the fact that those services were investigated on a user study basis only, none of the solutions made the step to integrate risk-related information from more than one source.

In the following we present the design, proceeding, and results from a survey we conducted in order to evaluate the suitability of using mobile phones as an interface to provide risk-related information.

\section{METHODOLOGY}

As described in 2, we base our methodological approach on the technology acceptance model which is applicable when dealing with mature technologies. Although the mobile phone can be regarded as a mature technology, a description of the mobile service which is subject to investigation is commonly provided. In order to give the participants of the survey a clearer understanding of the proposed service, graphical mock-ups were generated, describing the basic functionality (see Figure 1).

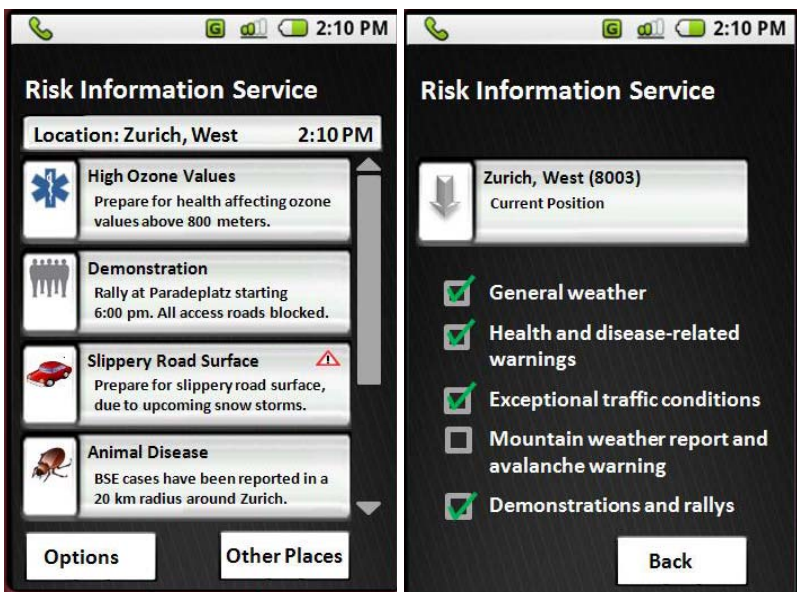

Figure 1. Risk information service mock-ups.

\subsection{Survey Design}

The survey was composed out of three parts: The collection of information about demographic factors (1), the investigation of the usefulness and intention to use the risk information service (2), and the evaluation of different design criteria (3):

(1) Besides the standard demographic factors age and gender, additional factors were investigated due to the context of mobile services. Besides the participant's mobile phone usage habits, their technology affinity and risk affinity were measured by adopting a set of proven constructs [9], [10].

(2) In order to evaluate the usefulness of the different aspects of the application as well as the participant's intention to use the application later on, we referred to a frame of questions commonly used in technology acceptance research for mobile services [11]. Based on this framework adjustments had to be made - especially concerning the evaluation of the perceived usefulness. We identified four aspects which defined the benefits of using the mobile phone as an interface to provide risk-related information:

- Location Dependency: Information provision about risks in the user's environment (e.g via GPS or CellID).

- Timely Notification: Timely provision of information due to permanent presence of the phone with the user.

- Risk Information Aspect: The fact that information about threats and risks is being provided in general.

- Customized Information: Possibility to select a set of risk related information which is relevant to the user.

Furthermore, questions were asked concerning the perceived influence of cost on the provision of such a service, the ease of use, and the participant's intention to actually use the service. Finally, the assumed usage frequency was investigated.

(3) In order to reach a high level of acceptance we regarded different design criteria for the mobile service. The user's preference concerning the delivery medium (mobile application, SMS, etc.) and the delivery mechanism (push vs. pull) were evaluated.

\subsection{Procedure}

Based on the design of the survey an online questionnaire was composed including mock-ups of the application in order to increase the participant's understanding of how the application could look like later on (see Figure 1). The questionnaire contained a total of 48 questions.

An email along with an invitation to the online survey was sent to an email list which contained 4087 recipients - most of them with a research affiliation which participated in the Internet of Things Conference 2008. The survey was accessible for 14 days from the 19.11.2008 to the 2.12.2008. A total of 308 respondents accessed the questionnaire whereof 130 completed the survey (Response rate $7.53 \%)$.

\section{RESULTS}

In the following the results from the survey are presented focusing on the demographic influence factors (4.1), the perceived usefulness and intention to use the application (4.2), and the evaluation of the design criteria (4.3).

\subsection{Demographics}

Based on the evaluation of the 130 complete responses $82 \%$ of the participants were male and $18 \%$ were female. Considering the age of the participants, 37\% were between 20 and 29, 25\% between 30 and 39, 20\% between 40 and $49,15 \%$ between 50 and 59 , and $3 \%$ above 59 (mean $=36.5$, standard deviation $=11.2)$. Most of the participants were using their mobile phones on a frequent basis: $46 \%$ stated to use their mobile phone very frequent, $34 \%$ frequent, $17 \%$ occasionally, and $3 \%$ rarely or never.

In addition to the basic demographic factors the participant's technology and risk affinity was collected. While $83 \%$ of the participants stated not to avoid new technologies and 82\% reported to be frequently asked about new technologies by others, only $35 \%$ confirmed to be among the first to buy products with 
new technological features. Concerning the risk attitude a slight tendency towards risk affine behavior among the participants could be noted: $8 \%$ strongly agreed and $37 \%$ agreed to engage in activities fraught with risk while on the other side $7 \%$ strongly rejected and $30 \%$ rejected to engage in such activities; $18 \%$ were undecided. The participant's affinity towards new technologies as well as the bias towards male participants reflects the nature of the survey sample which mostly consisted of researchers in the field of ubiquitous computing.

\subsection{Usefulness and Acceptance}

In order to evaluate the suitability of using mobile phones as an interface to provide risk-related information we asked the participants to rate the different features of such a service. The fact that risk-related information in general was provided was perceived useful by $62 \%$ of the participants. With the possibility to customize the notification about specific risks or events in mind, e.g. via a simple menu, $69 \%$ agreed that the possibility to receive information related to a certain type of event was useful. Due to the fact that the mobile phone constantly resides in our proximity the possibility to notify a user in time to react was evaluated. Here, $69 \%$ agreed that the timely provision of riskrelated information on the mobile phone was perceived as useful. Furthermore, the possibility to provide information about risks in the user's proximity, e.g. by locating him via GPS or Cell-ID, was investigated. The location dependent provision of risk-related information was perceived useful by $75 \%$ of the participants (Figure 2).

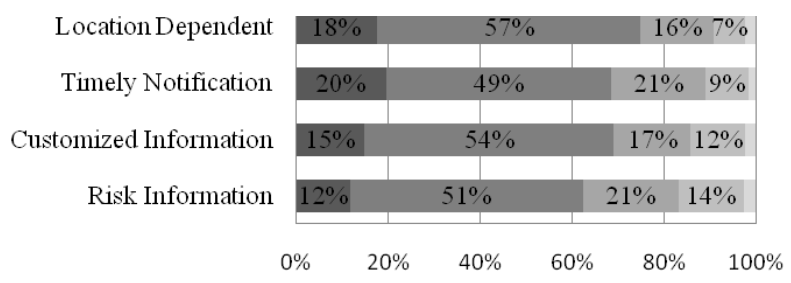

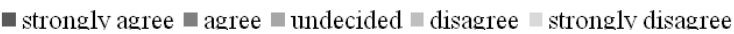

Figure 2. Interface inherent factors explaining the usefulness.

In addition to the factors which described the usefulness of the application, the ease of use and handling of the service was investigated. Based on the description and the mock-ups, $87 \%$ of the participants reported to perceive the application as easy to handle and configure.

Furthermore, the participants were asked to estimate the costs which would go along with the provision of such a service. While $59 \%$ preferred to buy the application once and use it without any further limitations, $49 \%$ still preferred to use the application on a pay-per-use basis, and only $19 \%$ preferred to pay for the usage on a monthly basis (Figure 3).

Finally, the participant's intention to use the service was evaluated: When asked for their intention to use the service $26 \%$ responded to use the service in the near future while $30 \%$ were undecided and $44 \%$ did not intend to use it.

When being asked to estimate their usage frequency of the service, two groups could be observed: $75 \%, 76 \%$, and $80 \%$ stated to use the service on a regular daily, weekly or monthly basis. In contract to that $55 \%$ responded to rather use the service on an irregular basis (Figure 4).

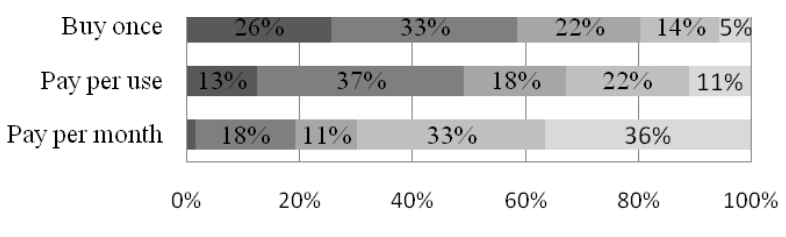

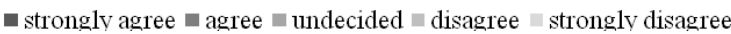

Figure 3. Influence of perceived cost.

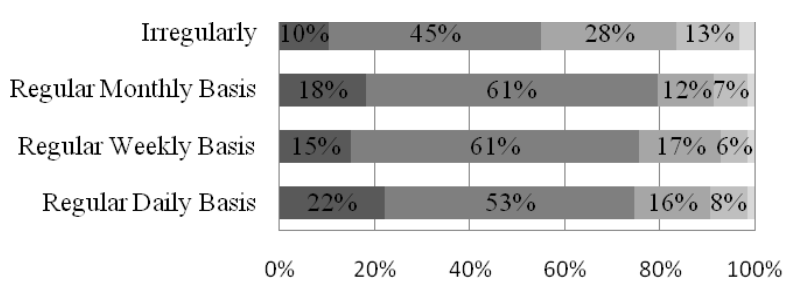

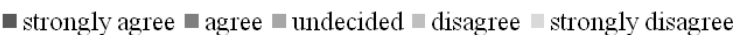

Figure 4. Intended usage behavior.

\subsection{Design Criteria}

Concerning the design of the service the preference for possible notification mechanisms and mediums which are feasible when providing information through the mobile phone were investigated: $66 \%$ of the participants responded to perceive the possibility of getting notified automatically (information push) as useful, while at the same time $67 \%$ perceived the possibility to request information manually at a given point of time (information pull) as useful (Figure 5). Finally, the participants were asked to rate different mediums by which the information could be provided. While only $6 \%$ preferred to get notified via a call agent, $46 \%$ preferred a notification via SMS/MMS, and $41 \%$ preferred to be notified by a separate application on the mobile phone (Figure 6).

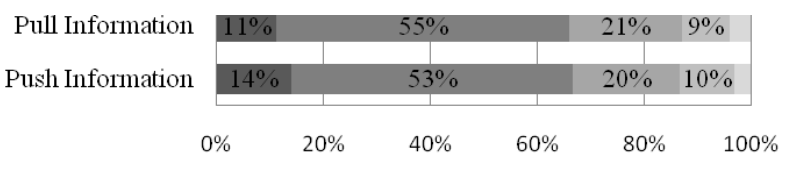

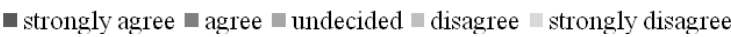

Figure 5. Preferred notification mechanism.

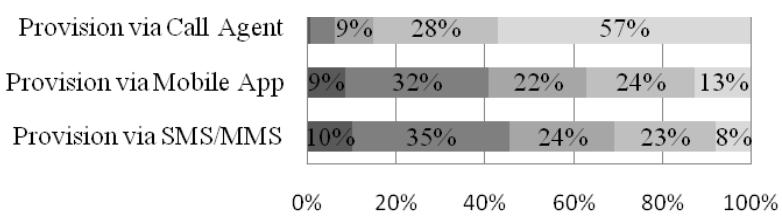

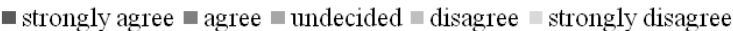

Figure 6. Preferred notification medium.

\section{DISCUSSION}

With the idea in mind that information about health, disease or environmental conditions is increasingly becoming available, we investigated the suitability of using mobile phones as an interface to provide risk-related information to the user. We approached the 
problem by conducting an online survey in order to investigate the acceptance, design alternatives, as well as operational issues.

(1) On a conceptual level we matched the requirements for a risk information service with the capabilities of the mobile phone. The requirements to provide risk-related information timely and tailored to the user's needs and location could be met when employing the mobile phone as an interface to provide this information. The aspects of timely, location dependent, and customized risk information provision were all perceived as useful by $67 \%$ to $75 \%$ of the participants (see Figure 2). Only a moderate acceptance could be proven with $25 \%$ of the participants intending to use the service and $44 \%$ not intending to use it. In addition to commonly quoted advantages of providing information via the mobile phone [4], we argue that the mobile phone offers a rich set of opportunities to provide risk-related information but requires further investigation as well.

(2) On a design level we investigated notification alternatives. Both, the automatic provision of information as well as the possibility to manually request information were perceived useful elements of a service providing risk-related information and should hence be part of the service (see Figure 5). In addition different notification mediums were considered. With the participants having rated equally important the provision of information via SMS/MMS and via a separate application, the development of a separate application poses an interesting opportunity. Due to the similar acceptance rates, providers of risk information services can overcome the functional and design limitations of SMS/MMS and include value-added services in the application (see Figure 6).

(3) On an operational level the influence of costs and the usage frequency were evaluated. The diverse interest of the participants to use the application on a regular and irregular basis implies that the application has to be tailored to the needs of two user segments. While the regular users might e.g. be more interested in an active notification about risk-related events, the irregular users might make use of the application in special situations only. The fact that about half of the participants assume that the provision of a risk information service goes along with costs (see Figure 3) either on a buy-once or pay-per-use basis - provides additional insights on the user's perception of this service. The willingness to pay for such a service, allows operators on the one hand to further investigate the purchasing behavior. On the other hand the willingness to pay confirms the perceived value in case the application is offered as a value-added service, as e.g. by insurance companies or travel agencies.

We have investigated the suitability of employing the mobile phone as an interface to provide risk-related information. The requirements such as the timely and location dependent provision of risk-related information could be met by the mobile phone and were perceived as useful by the participants of the survey. The participant's willingness to pay for the service on a per-use or monthly basis as well as the different regular and irregular user types provide valuable insights on the usage of the proposed service.

\section{CONCLUSION}

We have evaluated the potential of using mobile phones as an interface in order to provide risk-related information based on a survey on the user's acceptance. Furthermore, insights have been gathered on successful notification mechanisms and requirements such as the timely and location dependent provision of riskrelated information. Finally the participant's willingness to pay for the service on a per-use or monthly basis as well as the different regular and irregular user types provide valuable insights on the usage of the proposed service. With various fields of application in mind, our future research agenda includes the development of a prototype of the described risk information service. Along with a corporate partner from the insurance business we then plan to evaluate the usage behavior of the application when being provided as a value-added service.

\section{REFERENCES}

[1] Zhou, J., De Roure, D. 2007. FloodNet: Coupling adaptive Sampling with Energy Aware Routing in a Flood Warning System. Journal of Computer Science \& Technology. 22, 1, $121-136$

[2] Landry, B., Pierce, J., and Isbell, C. 2005. Supporting routine decision-making with a next-generation alarm clock. Persuasive Ubiquitous Computing. 8, 154 - 160.

[3] Signer, B., Norrie, M., Geissbuehler, P., and Heiniger, D. 2002. Telephone Interface for Avalanche Warnings based on Information Server for Adaptable Content Delivery. International Conference on Pervasive Computing. 725 740 .

[4] Keshav, S. 2005. Why Cell Phones Will Dominate the Future Internet. ACM SIGCOMM Computer Communication Review, 35.

[5] Venkatesh, V. 2003. User Acceptance of Information Technology: Towards a Unified View. MIS Quartely. 27, 3, 425-478.

[6] Abowd, G., and Mynatt, E. 2000. Charting Past, Present and Future Research in Ubiquitous Computing. ACM Transactions on Computer-Human Interaction. 7, 1, 29-58.

[7] Kaasinen, E. 2005. User acceptance of location-aware mobile guides based on seven field studies. Behaviour \& Information Technology. 24, 1, 37 - 49.

[8] Bina, M., and Giaglis, G. 2005. Exploring Early Usage Patterns of Mobile Data Services. Proceedings of the International Conference on Mobile Business 05. 363-369.

[9] Weber, E., Blais, A.-R., and Betz, N. 2002. A domainspecific risk-attitude scale: measuring risk perceptions and risk behaviors. Journal of Behavioral Decision Making. 15, 4, $263-290$.

[10] Parasuraman, A. 2000. Technology Readiness Index (Tri). Journal of Service Research, 2, 4, 307 - 320.

[11] Nysveen, H., Pedersen, P., and Thorbjørnsen, H. 2005. Intentions to use mobile services: Antecedents and crossservice comparisons. Journal of the Academy of Marketing Science.33, 3, $330-346$. 